\title{
Supporting Information:-
}

\section{An Enhanced PEC Water Splitting Performance of Silver Nanoparticles Coated CdS Nanowires Photoanode: The Role of Silver Deposition}

\author{
Sara Saeed ${ }^{1}$, Hassan Sddique ${ }^{1}$, Rucheng Dai ${ }^{2 *}$, Junke Wang ${ }^{1}$, Asad $\mathrm{Ali}^{1}$, Chan $\mathrm{Gao}^{2}$, Xiaoping Tao ${ }^{2}$, Zhongping Wang ${ }^{2}$, Zejun Ding ${ }^{1}$ and \\ Zengming Zhang ${ }^{1 \& 2^{*}}$ \\ ${ }^{1}$ Department of Physics and CAS Key Laboratory of Strong-Coupled Quantum Matter Physics, University of Science and Technology of \\ China, Hefei, Anhui 230026, China \\ ${ }^{2}$ The Center of Physical Experiments, University of Science and Technology of China, Hefei 230026, China
}

*Corresponding authors: dairc@ustc.edu.cn, zjding@ustc.edu.cn and zzm@ustc.edu.cn

\section{Experimental detail}

Reagents and materials All chemicals are of analytical grade and no further purification. FTO $\left(\mathrm{F}: \mathrm{SnO}_{2}\right)$ was used as substrate for the growth of nanostructures.

\subsection{Fabrication of Ag@CdS NWs}

The FTO glass substrates were ultra-sonicated with DI water, ethanol and acetone, then dried under a nitrogen flow. CdS thin film was fabricated by chemical bath deposition method, utilizing a solution composed of DI water, cadmium acetate dihydrate and thiurea as reported ${ }^{1}$. A small amount of ammonia was used to balance $\mathrm{pH}$ of the solution. FTO glass was hanged inside the solution. The system was heated at 85 ${ }^{\circ} \mathrm{C}$ for 24 minutes with continuous stirring. A pale-yellow, uniform thin film of CdS flat appeared on the substrate. The obtained samples were annealed at $250{ }^{\circ} \mathrm{C}$ in air to improve the crystallinity of thin film. The CdS NWs were synthesized by hydrothermal method according to a previously published procedure ${ }^{2}$, with a few modifications. 1 mole of cadmium acetate dihydrate were mixed with 2 moles of L-cysteine in 40 $\mathrm{ml}$ Teflon lined autoclave. Then $18 \mathrm{ml}$ of ethylenine diamine and $2 \mathrm{ml}$ of DI water were added. The FTO substrate $(2 \mathrm{~cm} \times 2 \mathrm{~cm})$, deposited 
with CdS flat layer was kept inside Teflon lined stainless steel autoclave, which was kept on $180{ }^{\circ} \mathrm{C}$ for 24 hours. After cooling to room temperature, the samples were washed with DI water and ethanol several times, then dried at room temperature.

Silver nanoparticles were deposited on CdS NWs by radio frequency (RF) magnetron sputtering. The deposition rate was about $0.8 \AA$ per second and the deposition time was $30 \mathrm{~s}, 45 \mathrm{~s}$ and $60 \mathrm{~s}$, respectively. The substrate temperature was maintained $100{ }^{\circ} \mathrm{C}$ for all samples. RF power used was $100 \mathrm{~W}$ at a pressure of $1 \mathrm{~Pa}$. As prepared samples were annealed for one hour at $500{ }^{\circ} \mathrm{C}$ in argon atmosphere at the Ar flow rate of 45 sccm. The Ag decorated CdS NWs were named as Ag(30s)@CdS NWs, Ag(45s)@CdS NWs, Ag(60s)@CdS NWs, respectively.

\subsection{PEC Measurements}

PEC measurements were carried out in a quartz windowed photoelectrochemical cell, on the electrochemical workstation (CHI760E) equipped with a three-electrode configuration, i.e., the photoanode (FTO/Ag@CdS) as a working electrode, while saturated calomel electrode (SCE) and Pt electrode were used as reference electrode and counter electrode. The surface areas exposed to the electrolyte were fixed at $1 \mathrm{~cm}^{2}$. An aqueous solution containing a mixture of $0.25 \mathrm{M} \mathrm{Na} \mathrm{S}_{2} \mathrm{~S}$ and $0.35 \mathrm{M} \mathrm{Na}_{2} \mathrm{SO}_{3}$ was used as an electrolyte for PEC measurements. The incident light from a $300 \mathrm{~W}$ Xe lamp was filtered to match with AM 1.5 G spectrum, which was used to provide visible illumination to the NWs photoanode. The visible light power intensity was $100 \mathrm{~mW} / \mathrm{cm}^{2}$, as measured by a radiometer (OPHIR, Littleton, CO). Linear sweep voltmeter measurements under dark and light conditions were performed at potential range from $-0.4 \mathrm{mV}$ to $0.2 \mathrm{mV}$ verses $\mathrm{SCE}$ electrode, at a scan rate of $50 \mathrm{mV} / \mathrm{S}$. Electrochemical impedance spectroscopy (EIS) were conducted at an applied voltage of $0.33 \mathrm{~V}$ and frequency range $10^{-2}$ to $10^{4} \mathrm{~Hz}$.

The amounts of evolved of $\mathrm{H}_{2}$ were tested at room temperature to analyze the PEC performance of Ag@CdS nanowires. The pristine CdS NWs and Ag(45S)@CdS NWs electrodes were used as photoanode with an active area $1 \mathrm{~cm}^{2}$ exposed to the electrolyte solution. The experiments were carried out in a $300 \mathrm{ml}$ single compartment tandem cell (comprising the two electrode system), half filled with electrolyte, leaving about $100 \mathrm{ml}$ of head space. The system was purged with Ar prior to measurements to remove dissolved gasses. Vacuum pump was used to establish vacuum in the PEC cell. A solar simulator equipped with $150 \mathrm{~W}$ Xe lamp with power intensity $100 \mathrm{~mW}^{\mathrm{c}} \mathrm{cm}^{2}$ was used as light source. $\mathrm{H}_{2}$ was analyzed by gas chromatography (Techcomp GC-7900) equipped with a thermal conductivity detector (TCD), with Ar as the carrier gas. The $0.6 \mathrm{ml}$ of gas sample was taken out from the PEC system by syringe, at a time interval of 20 min and injected to the GC. The obtained data was compared and analyzed by external standard method.

\section{Characterizations}


X-ray diffraction (XRD) measurements were performed on a Rigaku smart diffractometer equipped with Cu Ka radiation. Size and morphology of samples were characterized by a field emission scanning electron microscope (SEM) (ZEISS Gemini SEM 500) and high-resolution transmission electron microscopy (HRTEM) (JEOL JEM-ARM 200F) with selected area electron diffraction (SAED) and energy dispersive xray spectroscopy (EDX). X-ray photoelectron spectroscopy (XPS) spectra were recorded on ESCALAB 250 with Mg Ka (1253.6 eV) as the Xray excitation source. Optical absorption spectra were recorded by a spectrophotometer (Shimadzu DUV-3700). Photoluminescence (PL) spectra were recorded with an integrated laser Raman system (LabRAM HR800, Jobin Yvon) with a confocal microscope, stigmatic spectrometer and a multichannel air- cooled CCD detector at an excitation wavelength of $325 \mathrm{~nm}$.

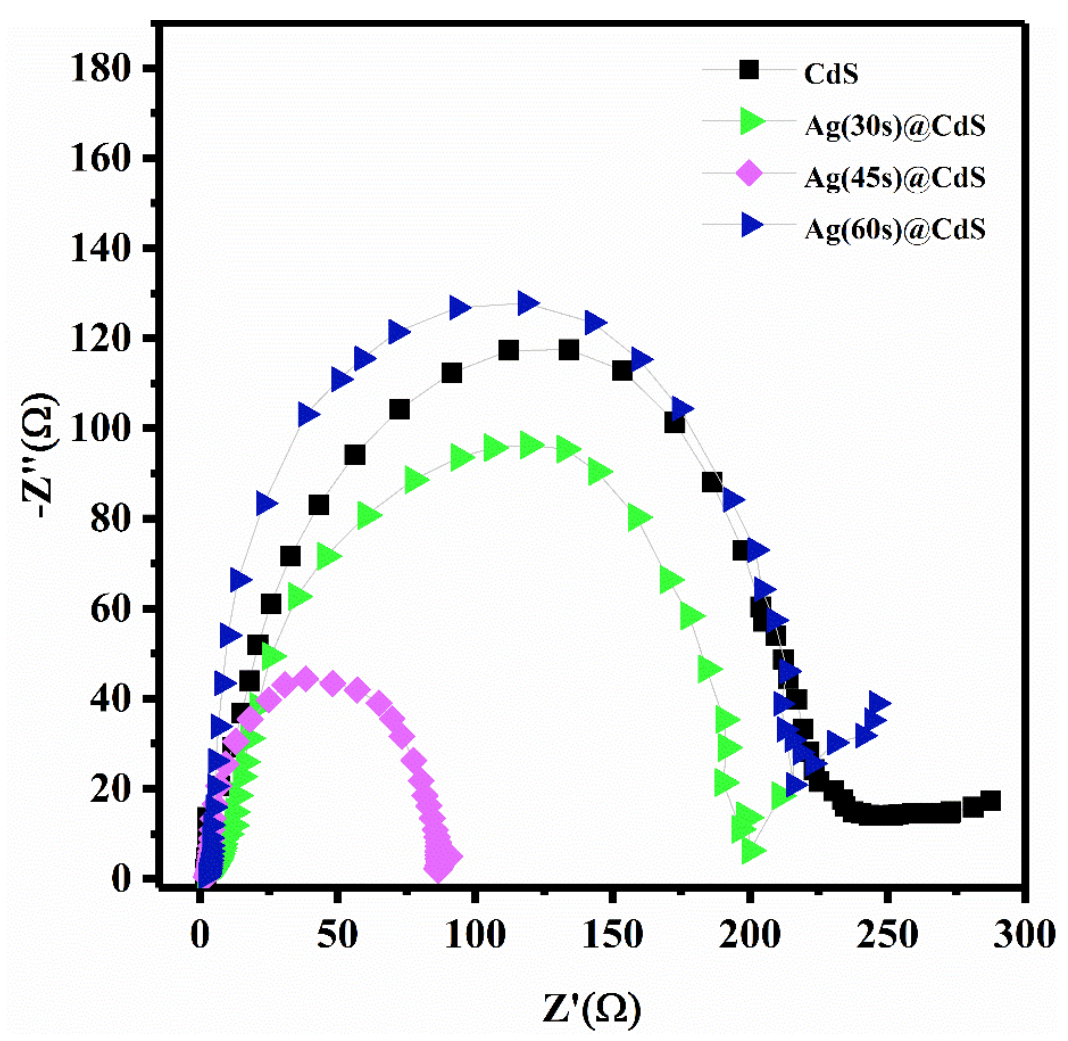

Figure S1. Electrochemical impedance spectra of Nyquist plots for, pure CdS, $\operatorname{Ag}(30 \mathrm{~s}) @ \mathrm{CdS}$, $\operatorname{Ag}(45 \mathrm{~s}) @ \mathrm{CdS}$ and $\mathrm{Ag}(60 \mathrm{~s}) @ \mathrm{CdS}$ nanowires under visible light irradiation. 
Table S1. Charge carrier density $\left(\mathrm{m}^{-3}\right)$ of pristine and Ag decorated samples calculated from the slope of mottschottky plots.

\begin{tabular}{cccc}
\hline $\mathrm{CdS}$ & $\mathrm{Ag}(30 \mathrm{~s}) @ \mathrm{CdS}$ & $\operatorname{Ag}(45 \mathrm{~s}) @ \mathrm{CdS}$ & $\operatorname{Ag}(60 \mathrm{~s}) @ \mathrm{CdS}$ \\
\hline $7.33 \times 10^{17}$ & $8.95 \times 10^{17}$ & $1.45 \times 10^{18}$ & $1.18 \times 10^{18}$ \\
\hline
\end{tabular}


Table S2. Photoelectrochemical water splitting performance of noble metal-CdS based hetro-structure photoanodes.

\begin{tabular}{|c|c|c|c|c|c|}
\hline Photoelectrode & $\begin{array}{l}\text { Electrolyt } \\
e\end{array}$ & $\begin{array}{l}\text { Light } \\
\text { source }\end{array}$ & $\begin{array}{l}\text { Photocurrent } \\
\text { density }\end{array}$ & $\begin{array}{l}\text { Photochemical } \\
\text { conversion efficiency } \\
\text { (PCE) \% } \\
\text { / hydrogen production }\end{array}$ & $\begin{array}{l}\text { Ref. } \\
\text { (year) }\end{array}$ \\
\hline $\begin{array}{l}P t / C d S / C o-P i \\
\text { nanorods }\end{array}$ & $\begin{array}{l}(0.25 \mathrm{M}) \\
\mathrm{Na}_{2} \mathrm{SO}_{4} \\
\text { and }(0.35 \\
\mathrm{M}) \mathrm{Na}_{2} \mathrm{~S}\end{array}$ & $\begin{array}{l}\text { AM } 1.5 \mathrm{G} \\
\text { illumination }\end{array}$ & $\begin{array}{l}5.05 \mathrm{mAcm}^{-2} \\
\text { at } 0.28 \mathrm{~V} \text { vs } \\
\text { RHE }\end{array}$ & ---- & $\begin{array}{c}3 \\
(2020)\end{array}$ \\
\hline $\begin{array}{l}\mathrm{ZnO} / \mathrm{CdS} / \mathrm{Au} \\
\text { nanotube arrays }\end{array}$ & $\begin{array}{l}(0.25 \mathrm{M}) \\
\mathrm{Na}_{2} \mathrm{~S} \text { and } \\
(0.35 \mathrm{M}) \\
\mathrm{Na}_{2} \mathrm{SO}_{3}\end{array}$ & $\begin{array}{l}350 \mathrm{~W} X \mathrm{Xe} \\
\text { lamp } \\
(\lambda>420 \\
\mathrm{nm})\end{array}$ & $\begin{array}{l}21.53 \mathrm{mAcm}^{-2} \\
\text { at } 1.2 \mathrm{~V} \mathrm{vs} \\
\mathrm{Ag} / \mathrm{AgCl}\end{array}$ & $\begin{array}{l}\text { PCE } 3.45 \% \\
\text { effective device area } \\
(1.5 \times 1.5) \mathrm{cm}^{2}\end{array}$ & $\begin{array}{c}4 \\
(2017)\end{array}$ \\
\hline $\begin{array}{l}\text { CeO2@Ag@CdS } \\
\text { nanotube arrays }\end{array}$ & $\begin{array}{l}(0.43 \mathrm{M} \\
) \mathrm{Na}_{2} \mathrm{~S} \text { and } \\
(0.5 \mathrm{M} \\
) \mathrm{Na}_{2} \mathrm{SO}_{3}\end{array}$ & $\begin{array}{l}300 \mathrm{~W} \text { Xe } \\
\text { lamp }\end{array}$ & $\begin{array}{l}2.14 \mathrm{~mA} \mathrm{~cm}^{-2} \\
(-0.2 \mathrm{~V} \text { (vs. } \\
\mathrm{Ag} / \mathrm{AgCl})\end{array}$ & ---- & $\begin{array}{c}5 \\
(2015)\end{array}$ \\
\hline $\begin{array}{l}\text { Au nanoparticle- } \\
\text { embedded } \\
\mathrm{ZnO} / \mathrm{CdS} \\
\text { core/shell } \\
\text { heterostructures }\end{array}$ & $\begin{array}{l}\mathrm{Na} 2 \mathrm{~S} \\
(0.25 \mathrm{M}) \\
\text { and } \\
\mathrm{Na}_{2} \mathrm{SO}_{3} \\
(0.35 \mathrm{M})\end{array}$ & $\begin{array}{l}150 \mathrm{~W} \text { Xe } \\
\text { lamp }\end{array}$ & $\begin{array}{l}<3.5 \mathrm{mAcm}^{-2} \\
\text { at } 0 \mathrm{~V} \text { versus } \\
\mathrm{Ag} / \mathrm{AgCl}\end{array}$ & PCE $3.2 \%$ & $\begin{array}{c}6 \\
(2020)\end{array}$ \\
\hline $\begin{array}{l}\text { Au@CdS } \\
\text { nanoparticles } \\
\text { modified } \mathrm{ZnO} \\
\text { nanowires }\end{array}$ & $\begin{array}{l}(0.25 \mathrm{M}) \\
\mathrm{Na}_{2} \mathrm{~S} \text { and } \\
0.35 \mathrm{M} \\
\mathrm{Na}_{2} \mathrm{SO}_{3}\end{array}$ & $\begin{array}{l}150 \mathrm{~W} \text { Xe } \\
\text { lamp }\end{array}$ & $\begin{array}{l}2.5 \mathrm{mAcm}^{-2} \\
\text { vs } \mathrm{Ag} / \mathrm{AgCl}\end{array}$ & $\begin{array}{l}\mathrm{H}_{2}=11.5 \mu \mathrm{mol} \mathrm{h}^{-1} \\
\text { (Active device } \\
\text { area }=1.2 \mathrm{~cm}^{2} \text { ) }\end{array}$ & $\begin{array}{c}7 \\
(2015)\end{array}$ \\
\hline $\begin{array}{l}\text { CdS } / A g / Z n O \\
\text { Nanorods }\end{array}$ & $\begin{array}{l}(0.25 \mathrm{M}) \\
\mathrm{Na}_{2} \mathrm{~S} \text { and } \\
(0.35 \mathrm{M}) \\
\mathrm{Na}_{2} \mathrm{SO}_{3}\end{array}$ & $\begin{array}{l}500 \mathrm{~W} \text { Xe } \\
\text { lamp }\end{array}$ & $\begin{array}{l}\sim 4 \mathrm{mAcm}^{-2} \text { at } \\
0 \mathrm{~V} \text { vs SCE }\end{array}$ & $\begin{array}{l}\text { PCE } 3.13 \% \\
\left(\text { active area }=1 \mathrm{~cm}^{2}\right)\end{array}$ & $\begin{array}{c}8 \\
(2017)\end{array}$ \\
\hline $\begin{array}{l}\text { Hollow CdS } \\
\text { nanoprisms }\end{array}$ & $\begin{array}{l}(0.5 \mathrm{M}) \\
\mathrm{Na}_{2} \mathrm{~S} \text { and } \\
(0.5 \mathrm{M}) \\
\mathrm{Na}_{2} \mathrm{SO}_{3}\end{array}$ & $\begin{array}{l}300 \mathrm{~W} \text { Xe } \\
\text { lamp }\end{array}$ & $\begin{array}{l}1.5 \mathrm{mAcm}^{-2} \text { at } \\
0 \mathrm{~V} \mathrm{Vs} \\
\mathrm{Ag} / \mathrm{AgCl}\end{array}$ & $\begin{array}{c}\text { PCE } 0.80 \% \\
\mathrm{H}_{2}=46.7 \mu \text { mol after } \\
3 \mathrm{hr} \text { illumination } \\
\left(\text { active area }=1 \mathrm{~cm}^{2}\right)\end{array}$ & $\begin{array}{c}9 \\
(2019)\end{array}$ \\
\hline $\begin{array}{l}\text { Ag@CdS } \\
\text { nanowires }\end{array}$ & $\begin{array}{l}(0.25 \mathrm{M}) \\
\mathrm{Na}_{2} \mathrm{~S} \text { and } \\
(0.35 \mathrm{M}) \\
\mathrm{Na}_{2} \mathrm{SO}_{3}\end{array}$ & $\begin{array}{l}300 \mathrm{~W} X \mathrm{Xe} \\
\operatorname{lamp} \\
(\lambda>420 \\
\mathrm{nm})\end{array}$ & $\begin{array}{l}6.15 \mathrm{mAcm}^{-2} \\
\text { at } 0.18 \mathrm{~V} \mathrm{vs} \\
\text { SCE }\end{array}$ & $\begin{array}{l}\quad \text { PCE } 6.6 \% \\
\mathrm{H}_{2}=129.2 \mu \text { mole after } \\
4 \mathrm{hr} \text { illumination } \\
\left(\text { active area }=1 \mathrm{~cm}^{2} \text { ) }\right.\end{array}$ & This work \\
\hline
\end{tabular}




\section{References}

(1) Bai, Z.; Wan, L.; Hou, Z.; Wang, D. Effect of $\mathrm{CdCl}_{2}$ Annealing Treatment on CdS Thin Films and CdTe/CdS Thin Film Solar Cells. Phys. Status Solidi Curr. Top. Solid State Phys. 2011, 8 (2), 628-630.

Ren, B.; Cao, M.; Zhang, Q.; Huang, J.; Zhao, Z.; Jin, X.; Li, C.; Shen, Y.; Wang, L. Controllable Synthesis of CdS Nanowire by a Facile Solvothermal Method and Its Temperature Dependent Photoluminescent Property. J. Alloys Compd. 2016, 659, 74-81.

Ruan, M.; Cai, X.; Lan, Y.; Xing, H. Hexagonal CdS Photoanode Modified with Pt and Cobalt Phosphate Cocatalyst for Efficient Photoelectrochemical Performance. Mater. Lett. 2020, 260, 126947.

Wei, R. Bin; Kuang, P. Y.; Cheng, H.; Chen, Y. B.; Long, J. Y.; Zhang, M. Y.; Liu, Z. Q. Plasmon-Enhanced Photoelectrochemical Water Splitting on Gold Nanoparticle Decorated ZnO/CdS Nanotube Arrays. ACS Sustain. Chem. Eng. 2017, 5 (5), 4249-4257.

Zhao, M.; Li, H.; Shen, X.; Ji, Z.; Xu, K. Facile Electrochemical Synthesis of $\mathrm{CeO}_{2} @ \mathrm{Ag} @ \mathrm{CdS}$ Nanotube Arrays with Enhanced Photoelectrochemical Water Splitting Performance. Dalt. Trans. 2015, 44 (46), 19935-19941.

Hieu, H. N.; Nghia, N. V.; Vuong, N. M.; Van Bui, H. Omnidirectional Au-Embedded ZnO/CdS Core/Shell Nanorods for Enhanced Photoelectrochemical Water-Splitting Efficiency. Chem. Commun. 2020, 56 (28), 3975-3978.

Yang, X.; Li, H.; Zhang, W.; Sun, M.; Li, L.; Xu, N.; Wu, J.; Sun, J. High Visible Photoelectrochemical Activity of Ag NanoparticleSandwiched CdS/Ag/ZnO Nanorods. ACS Appl. Mater. Interfaces 2017, 9 (1), 658-667. 